\title{
Stop the leak!: Mitigating potential exposure of aerosolized COVID-19 during laparoscopic surgery
}

\author{
John M. Uecker ${ }^{1,3} \cdot$ Austin Fagerberg $^{1} \cdot$ Naser Ahmad $^{1} \cdot$ Alexander Cohen $^{2} \cdot$ Mitchell Gilkey $^{1} \cdot$ Farshid Alembeigi $^{2}$. \\ Christopher R. Idelson ${ }^{1}$ (i)
}

Received: 2 July 2020 / Accepted: 16 September 2020 / Published online: 24 September 2020

○) Springer Science+Business Media, LLC, part of Springer Nature 2020

\begin{abstract}
Background Viral particles have been shown to aerosolize into insufflated gas during laparoscopic surgery. In the operating room, this potentially exposes personnel to aerosolized viruses as well as carcinogens. In light of circumstances surrounding COVID-19 and a concern for the safety of healthcare professionals, our study seeks to quantify the volumes of gas leaked from dynamic interactions between laparoscopic instruments and the trocar port to better understand potential exposure to surgically aerosolized particles.

Methods A custom setup was constructed to simulate an insufflated laparoscopic surgical cavity. Two surgical instrument use scenarios were examined to observe and quantify opportunities for insufflation gas leakage. Both scenarios considered multiple configurations of instrument and trocar port sizes/dimensions: (1) the full insertion and full removal of a laparoscopic instrument from the port and (2) the movement of the scope within the port, recognized as "dynamic interaction", which occurs nearly $100 \%$ of the time over the course of any procedure.

Results For a $5 \mathrm{~mm}$ instrument in a $5 \mathrm{~mm}$ trocar, the average volume of gas leaked during dynamic interaction and full insertion/removal scenarios were 43.67 and $25.97 \mathrm{~mL}$ of gas, respectively. Volume of gas leaked for a $5 \mathrm{~mm}$ instrument in a $12 \mathrm{~mm}$ port averaged $41.32 \mathrm{~mL}$ and 29.47 for dynamic interaction vs. instrument insertion and removal. Similar patterns were shown with a $10 \mathrm{~mm}$ instrument in $12 \mathrm{~mm}$ port, with $55.68 \mathrm{~mL}$ for the dynamic interaction and 58.59 for the instrument insertion/removal.

Conclusions Dynamic interactions and insertion/removal events between laparoscopic instruments and ports appear to contribute to consistent leakage of insufflated gas into the OR. Any measures possible taken to reduce OR gas leakage should be considered in light of the current COVID-19 pandemic. Minimizing laparoscope and instrument removal and replacement would be one strategy to mitigate gas leakage during laparoscopic surgery.
\end{abstract}

Keywords Laparoscopy $\cdot$ Insufflation $\cdot$ Coronavirus $\cdot$ COVID-19 $\cdot$ Exposure $\cdot$ Operating room

Clinicians working on the frontlines have continued to adapt to the ever-changing environment that is living and working

John M. Uecker, Farshid Alembeigi, and Christopher R. Idelson have contributed equally to this work.

Christopher R. Idelson

cidelson@clearcam-med.com

1 ClearCam Inc, Austin, TX 78744, USA

2 Department of Mechanical Engineering, The University of Texas at Austin, Austin, TX 78712, USA

3 Department of Surgery and Perioperative Care, Dell Medical School, The University of Texas at Austin, Austin, TX 78712, USA during the COVID-19 pandemic. Despite the many precautions that clinicians have taken against COVID-19, there are almost 10,000 healthcare workers that have been diagnosed with coronavirus as of April 17th, 2020, and at least half of these were contracted through patient contact [1].

In light of these historic events, many surgical organizations including the Society of American Gastrointestinal and Endoscopic Surgeons (SAGES), the European Association of Endoscopic Surgery (EAES), the American College of Surgeons (ACS), and many others have issued recommendations to postpone elective surgeries [2-8]. In addition to these recommendations, SAGES encourages the use of minimally invasive surgery and robotic surgery techniques whenever possible. Implementing minimally 
invasive surgical techniques help reduce hospital stay time for patients, allowing a greater capacity of empty beds for the treatment of COVID-19 while simultaneously minimizing clinician exposure to potentially contagious patients [2]. Extreme intervention efforts are being made in the medical community to mitigate healthcare worker exposure to the contagious virus, with notable focus on exposure in the Operating Room (OR) [9].

\section{Aerosolized viral particles in surgery}

While the use of minimally invasive surgery is thought to mitigate the exposure of clinicians to contagious patients compared to other clinical settings, it is not without risk! Greater concentrations of coronavirus particles have been found in peritoneal fluid than in the respiratory tract, which demonstrates a previously unknown risk to surgeons and their staff during surgery [10]. The laparoscopic environment also lends itself to the aerosolization of tissue particles. Specifically, the use of certain laparoscopic instrumentation such as the ultrasonic scalpel, electrocautery, and laser ablation have been shown to aerosolize tissue particles into smoke during surgery [11]. Furthermore, active viral particles have been shown to aerosolize via the same mechanism. Even more concerning is the fact that this space is not yet well-understood or -explored, as few studies have been performed to understand this issue at levels sufficient enough to garner strong understanding and scope of the potential problem [11-17]. However, those few studies have shown that viruses such as Hepatitis B and HPV have been shown to aerosolize in the smoke evacuated during laparoscopic surgery as well as during open surgery [11, 18-25]. The known aerosolization of these other viruses combined with several new articles documenting COVID-19 in the peritoneal fluid have led numerous international surgical societies to encourage exercising caution when performing minimally invasive surgery [2-8].

In addition to evacuated smoke, there is evidence to suggest that leaked $\mathrm{CO}_{2}$ gas from the insufflation of the pneumoperitoneum could contain aerosolized virus particles [11]. This leaked $\mathrm{CO}_{2}$ may contain viral particles as shown in the previous studies, suggesting a serious concern to the health and safety of clinical professionals in the OR, especially given the growing concern regarding the potential aerosolization of coronavirus particles $[2,3,11,18,19,21$, $22,26]$. Due to the highly contagious nature of COVID-19, and our relative lack of understanding of its mode of transmission via aerosolization, Kwak et al. [18] further recommend that any measures taken to minimize gas leakage during laparoscopic surgery should be considered for the safety of operating room healthcare providers. Relevant studies of coronavirus concerning the aerosolization of COVID-19 and infection of surgeons or other medical staff through these aerosols remain relatively unexplored, but the issue continues to be a growing concern [15, 27-30]. Further support of this notion is the fact that both SAGES and the EAES strongly recommend the considered use of devices capable of ultra-filtration regarding released $\mathrm{CO}_{2}$ and smoke due to the concern for aerosolized COVID-19 particles [2].

As shown, even with limited data surrounding this problem, there is significant concern among surgical societies and operating rooms across the globe. Although data is limited, numerous studies suggest reducing exposure to laparoscopic gases in order to mitigate exposure of COVID-19, and other viruses, to operating room personnel [31-34]. As such, it is clear that more research is needed in these areas to help understand and quantify potential exposure concerns in the OR.

\section{Need for more research to accurately quantify the problem}

While these suggestions attempt to address concerns (primarily) regarding "controlled" gas leakage, there are a number of uncontrolled gas leak mechanisms that have been postulated by the medical community, yet also remain relatively unexplored, around the trocar port and through instrument changes. Early stage studies have shown prominent attention given to gas leakage through the trocar (port) [2, 35]. And no data currently exists in literature that attempts to quantify gas leakage during instrument use and exchange, despite the fact the literature shows that such events are overly abundant in every laparoscopic procedure [36-38]. More work is needed to better understand the level of risk of clinician safety in today's OR to guide us to a safer OR of the future around this rising issue, brought to the forefront of people's minds during this global COVID-19 pandemic. The proposed study focuses on better quantifying gas leakage regarding instrument-port related events in order to better understand potential risks to today's OR clinicians.

\section{Materials and methods}

IRB approval was not required for this study. The report details a test setup and results evaluating the gas leaked while simulating realistic scenarios happening during a laparoscopic surgery using two size trocar configurations, $5 \mathrm{~mm}$ and $12 \mathrm{~mm}$ trocar ports (Applied Medical Kii Balloon Blunt Tip System $12 \times 100 \mathrm{~mm} \&$ Applied Medical Kii Balloon Blunt Tip System 5×100 mm; Ranch Santa Margarita, CA), and two laparoscopic instruments, laparoscopes measuring 5 mm (Stryker 502-555-030; Kalamazoo, MI) and $10 \mathrm{~mm}$ (Richard Wolf 8934-442 Panoview; Vernon Hills, IL) each. 


\section{Test setup}

A photo of the benchtop test setup can be seen in Fig. 1 [39]. The setup uses a compressor (California Air Tools CAT-1P1060S; San Diego, CA) combined with a regulator (30222 Fairchild Model 30 Compact Precision Regulator; Winston-Salem, NC) and pressure gauge (General Electric Druck DPI 104-IS; Leicester, UK) to simulate a laparoscopic insufflator to maintain a consistent and reliable pressure. The regulator has an on/off valve that controls the flow of compressed air to a mass flow meter (Model FMA1820A; Norwalk, CT) that is used to measure the leak flow rate of the system and records data via a data logger.
Next, gas travels to an insufflation chamber, comprised of a plastic tube with an elastic seal at the proximal end to mimic compliant tissue through which a trocar port is placed for testing. Data were logged using a National Instrument NI4350 data logger. The insufflation chamber was made using a $1 \mathrm{ft}$ PVC tubing, on which the distal end was connected to a compliant rubber tubing from which to maintain insufflation pressure and monitor any insufflation gas leakage.

The simulated benchtop pressure-management system essentially performs the same function as a laparoscopic insufflation system, proving quite adequate as simulated OR equipment. The plastic tube of the insufflation cavity is rigid (unlike a patient's abdomen) and the thin elastic seal is notably compliant (though not quite as compliant as human tissue). While this is a shortcoming compared to testing in
Fig. 1 Physical benchtop and diagram outlining experimental setup and correlating setup in an OR environment

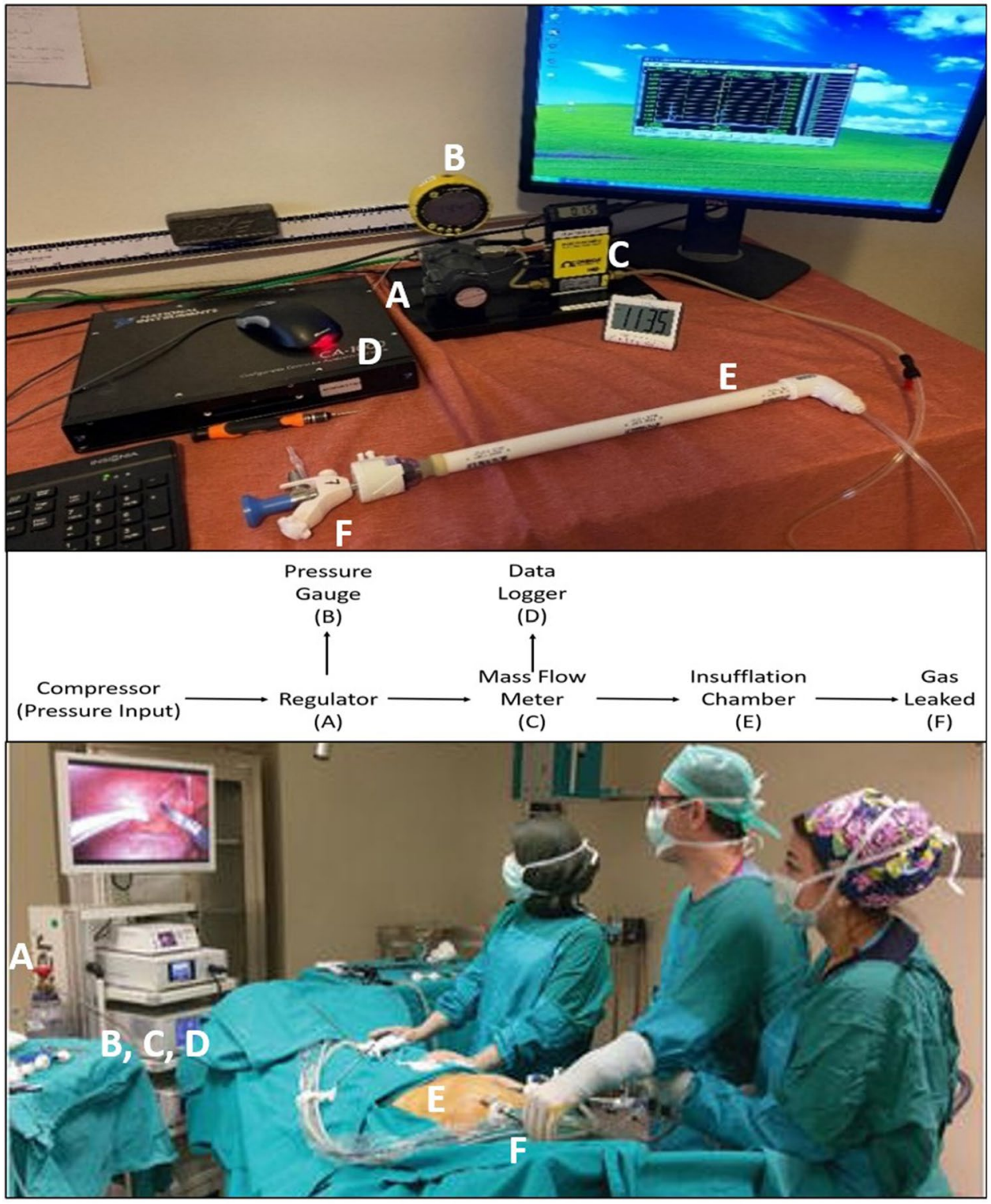


a real patient or cadaver model, the benchtop setup is still sufficient enough to yield functional results surrounding data trends. Limitations of the benchtop experiment could be improved in future iterations but suffice for this exploratory experiment that aims to determine potential health hazards for clinicians in today's OR.

Exploratory test configurations are outlined in Table 1 for instrument-port sizes and combinations. Shorthand for laparoscopic instrument levels are designated by " $L$ " followed by dimension (i.e. $5 \mathrm{~mm}$ instrument level henceforth known as L5 and $10 \mathrm{~mm}$ as L10). Shorthand for trocar port levels are designated by " $\mathrm{T}$ " followed by dimension (i.e. $5 \mathrm{~mm}$ trocar port level henceforth known at T5, and $12 \mathrm{~mm}$ as T12).

The desired trocar configuration was inserted into the elastic seal end of the PVC pipe. The full length of the trocar was inserted creating a seal around the inner dimeter port the outer diameter of the trocar stem, simulating a best-case scenario seen in laparoscopic surgery between the port and tissue. Once the desired configuration was setup up the following steps were followed for each clinical situation. A "null" (i.e. trocar-only) configuration was tested to understand potential trocar port or elastic seal-trocar port interaction leakage. Such data was used to confirm that the test setup was capable of sustaining pressure to ensure the test setup had no leaks before instrument-port interaction was initiated.

\section{Test procedure for each use case scenario}

Each configuration and use case included in this study was designed to simulate typical events observed in today's laparoscopic OR. The Null configuration scenario was used to

Table 1 Test configurations [39]

\begin{tabular}{lll}
\hline $\begin{array}{l}\text { Test } \\
\text { configu- } \\
\text { ration }\end{array}$ & $\begin{array}{l}\text { Instrument } \\
\text { size }(\mathrm{mm})\end{array}$ & $\begin{array}{l}\text { Port } \\
\text { size } \\
(\mathrm{mm})\end{array}$ \\
\hline 1 & 5 & 5 \\
2 & 5 & 12 \\
3 & 10 & 12 \\
\hline
\end{tabular}

simulate a trocar port placed in a patient without any instrument being used with that port at a specific time. While this configuration is not common during surgery (no human instrument or scope driver is often "perfectly still"), it might be representative in nature (though not in number) to robotic surgeries, where a robot is able to hold a scope near-perfectly still. This configuration also doubled as a baseline test for the experimental setup. The Dynamic Instrument-Port Interaction scenario represented a common use case where a clinician might be moving a laparoscope, cutters, cautery, or other such elongated instrument within a trocar to perform surgery. Essentially, in laparoscopic and robotic surgeries, instruments are nearly constantly moving and interacting with trocar ports/valves, suggesting that these results indicate a real-time representation of OR instrument usage. The Instrument Full Insertion/Removal scenario was used to simulate an instrument exchange or other such removal and/or insertion event, which is commonly experienced during laparoscopy for each and every case. For example, scope removals are often performed to clean the lens, and instruments are commonly removed to achieve access through a different port or to exchange them out for a new instrument [36, 37, 40-43]. Each instrument-port configuration also helped to represent a few different levels of such combinations seen in today's OR.

(1) Null configuration scenario:

1. Open valve and stabilize insufflation chamber pressure.

2. Rotate valve counterclockwise to the closed position to depressurize chamber.

3. Confirm stable pressure remains and no leaks are present in the test setup.

(2) Dynamic Instrument-Port Interaction scenario:

1. Insert laparoscopic instrument by $3 / 4$ of its full length through the port ( 9 inches) (Fig. 2).

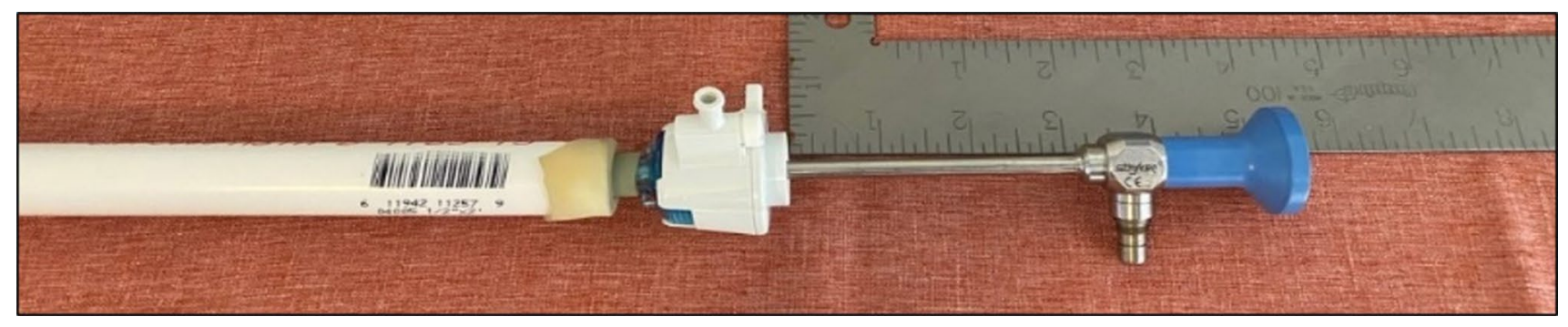

Fig. 2 Dynamic instrument-port interaction setup with 3 inch offset from full device insertion 
2. Open valve and stabilize insufflation chamber pressure.

3. Insert remaining partial length, approximately 3 inches, of the laparoscopic instument until it bottoms out on the trocar.

4. Wait approximately $10 \mathrm{~s}$ for pressure to stabilize.

5. Remove the instrument partially 3 inches.

6. Wait approximately $10 \mathrm{~s}$ for pressure to stabilize.

7. Repeat steps 3-6 two more times.

8. Wait $5 \mathrm{~s}$ for pressure to stabilize.

\section{(3) Instrument Full Insertion/Removal scenario:}

1. Open valve and stabilize insufflation chamber pressure.

2. Rotate valve counterclockwise to the closed position to depressurize chamber.

3. Fully insert the laparoscopic instrument through the port.

4. Wait approximately $10 \mathrm{~s}$ for pressure to stabilize.

5. Fully remove the laparoscopic instrument from the port.

6. Wait approximately $5 \mathrm{~s}$ for the pressure to stabilize.

\section{Results}

\section{Null configuration scenario}

$5 \mathrm{~mm}$ and $12 \mathrm{~mm}$ trocar ports were used for each configuration ( $n=1$ device and $n=3$ trials per configuration). Results confirm that both ports have negligible gas leaks when integrated with the test setup. The $5 \mathrm{~mm}$ port registered an average $0.02 \mathrm{~L} / \mathrm{min}$ leak rate, while the $12 \mathrm{~mm}$ port registered no leak. While the reason for the small $5 \mathrm{~mm}$ leak could be attributed to either (1) a manufacturing/device design trait or (2) a test setup interaction with the port, the result of a negligible leak remains consistent and the setup was shown to be highly reliable.

\section{Dynamic instrument-port interaction scenario}

The dynamic instrument-port interaction scenario involved three advancement and retraction events per trial and was performed for each instrument-port configuration ( $n=3$ trials per configuration). Figure 3 shows graphs displaying results for both individual and averaged

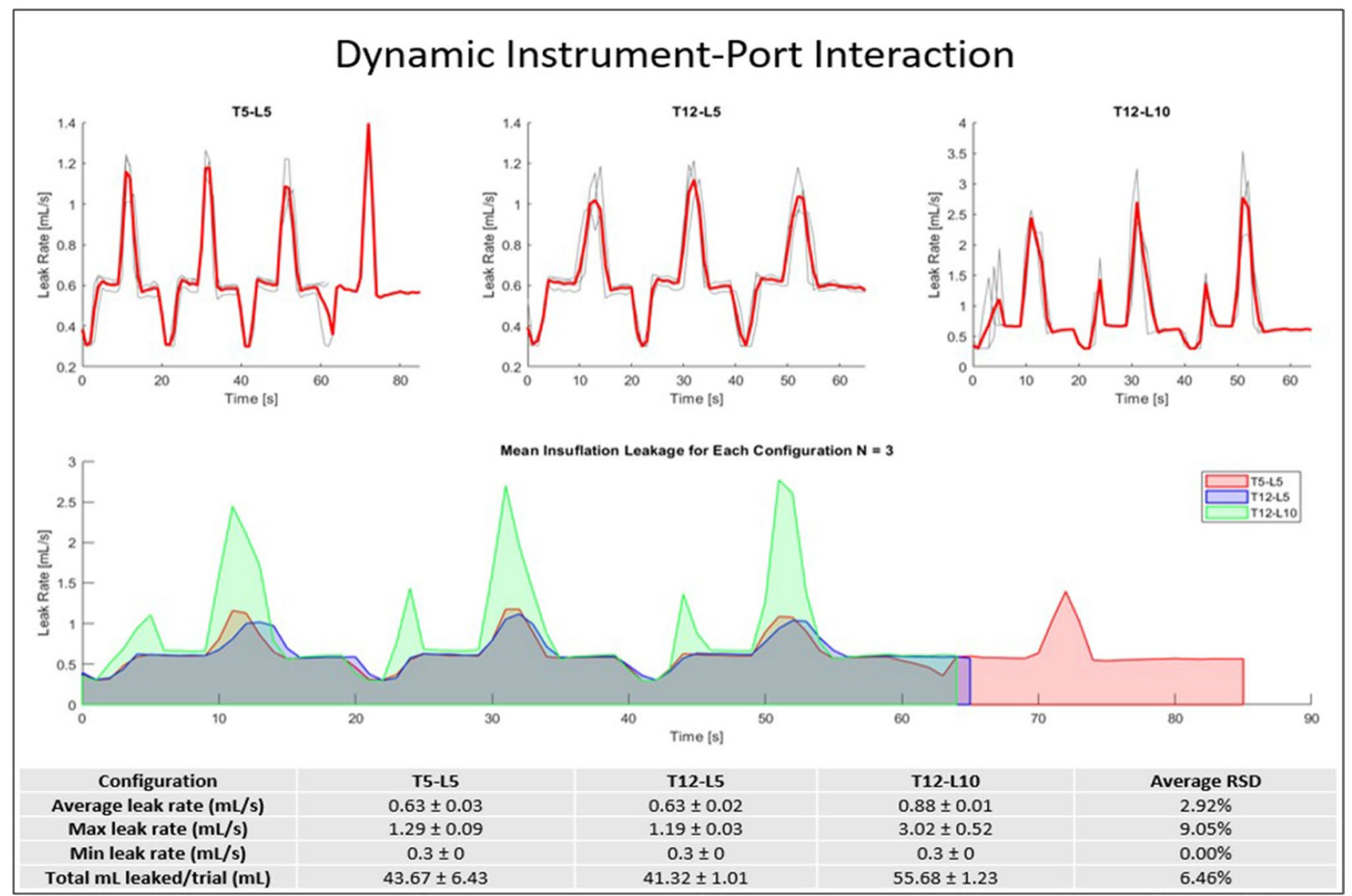

Fig. 3 Gas leaked during dynamic interaction of instrument-port devices. Best viewed in color 
trials. T5-L5 averaged $43.67 \mathrm{~mL}$ of leaked gas per trial while T12-L5 and T12-L10 averaged $41.32 \mathrm{~mL}$ and $55.68 \mathrm{~mL}$ of leaked gas, respectively.

\section{Instrument full insertion/removal scenario}

The laparoscopic instrument full insertion/removal scenario outlined above was performed for each instrument-port configuration, with additional trials performed in comparison with previous test configurations in an attempt to counteract additional noise variables experienced for this protocol ( $n=10$ trials per configuration). Graphs observed in Fig. 4 show results for individual and averaged trials. Test configuration combinations show different levels of gas leakage for simulated instrument insertion and removal events: T5-L5 $=25.97 \mathrm{~mL}$, $\mathrm{T} 12-\mathrm{L} 5=29.47 \mathrm{~mL}$, and $\mathrm{T} 12-\mathrm{L} 10=58.59 \mathrm{~mL}$.

\section{Discussion}

Insufflation leakage during laparoscopic surgery has become a prevalent topic of conversation in surgical societies around the globe in light of the COVID-19 outbreak. The data presented quantifies the amount of potential leakage that may be attributed to instrument interactions with the trocar port, including typical surgical dynamic use as well as insertion/ removal events during laparoscopic surgery.

Specifically, for the full insertion/removal scenario, once the instrument was inserted into the trocar flow rate began to increase after initial leakage from opening of the trocar port valve occurred, and then stabilizes with a stationary instrument. This suggests that a stationary instrument inside the trocar port still produces a constant gas leak. Then, as the instrument was removed, the flow rate appears to reach a peak, demonstrating a higher amount of gas leakage during dynamic port valve interaction with instrumentation (further supported by data from the Dynamic Instrument-Port Interaction protocol).

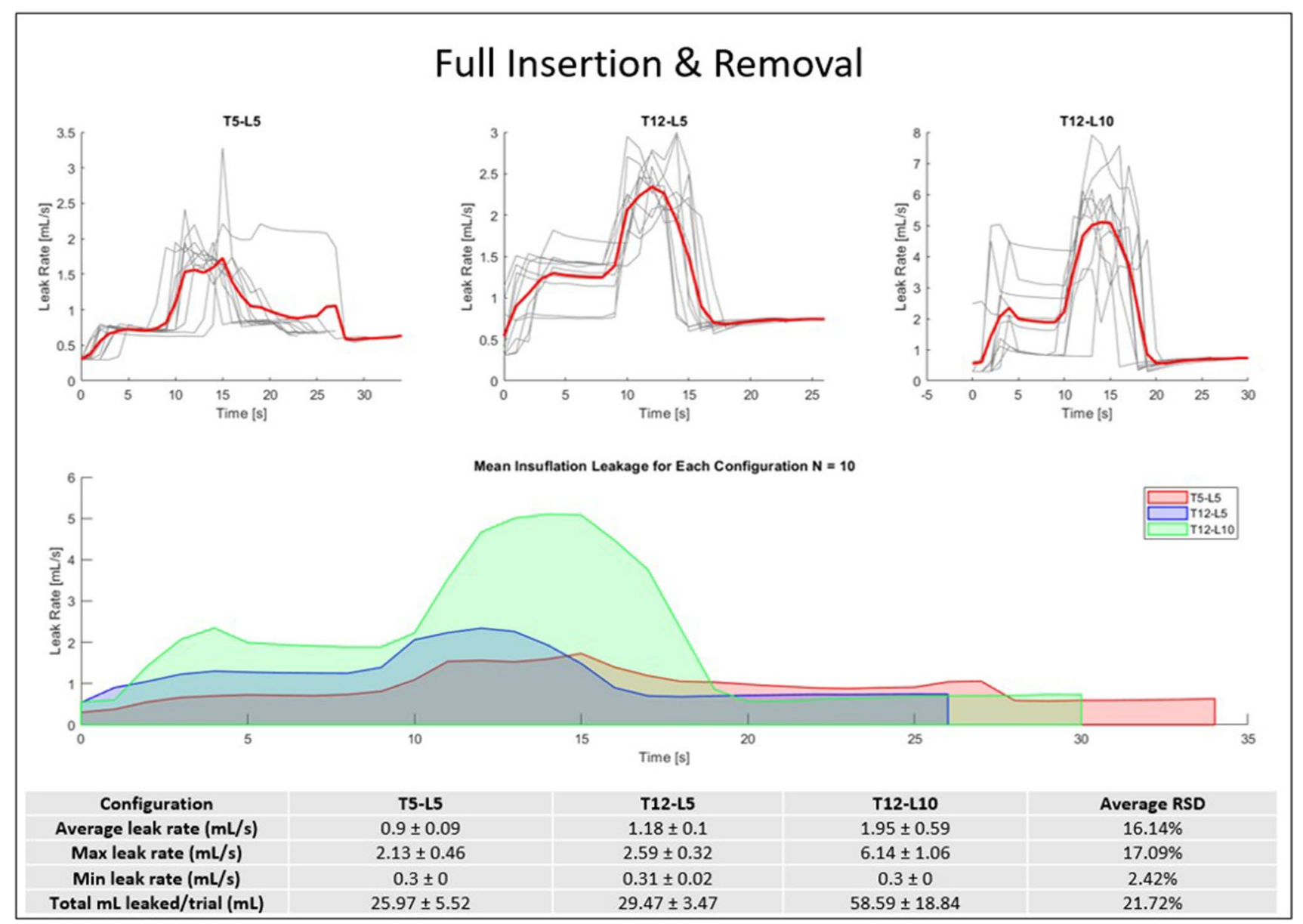

Fig. 4 Gas leaked during insertion and removal of a laparoscopic instrument through a trocar. Best viewed in color 
Table 2 Clinically translatable estimates

\begin{tabular}{llrr}
\hline Configuration & T5-L5 & T12-L5 & T12-L10 \\
\hline Estimated gas volume leaked in OR within 1 h $(\mathrm{mL})$ & Dynamic port-instrumentation interaction & \\
& 2160.00 & 2160.00 & 2880.00 \\
& Full insertion and removal & & \\
& 324.00 & 396.00 & 684.00 \\
\hline
\end{tabular}

After the instrument was fully removed, the flow rate then approaches its baseline as the trocar port valve is allowed to fully seal again since no instrumentation is present to keep it open. Data from this study show that instrument interaction with a trocar port, especially considering instrument-port dynamic interaction/motion as well as full instrument insertion and removal events, stands to produce gas leakage across $5 \mathrm{~mm}$ and $10 \mathrm{~mm}$ instruments in $5 \mathrm{~mm}$ and $12 \mathrm{~mm}$ trocars. Further work should consider additional levels of instrumentation-port interactions and configurations regarding different laparoscopic instrument motions and sizes/dimensions. Additionally, similar testing of instrument-port interactions in a clinical setting would more directly show the amount of gas leaked during a surgery from this leakage source and should further look to understand volumes of aerosolized viral particles exposed to the OR environment during surgery to better understand potential instances of exposure to airborne particulates. In addition, only "insufflation pressures" of $15 \mathrm{mmHg}$ were tested. This is a common pressure used during laparoscopic surgery [44]. Currently, recommendations from surgical societies such as SAGES that consider decreasing leakage include using lower intra-abdominal pressures when technically possible. It is possible that higher or lower insufflation pressures could affect results. Additionally, the test setup might benefit from additional alterations to more closely overcome the limitations discussed in 'Materials and methods' of this article.

Further extrapolation of the presented data can be considered for insufflation leakage estimated to be released during $1 \mathrm{~h}$ of laparoscopic surgery (Table 2). The dynamic port-instrumentation interaction over $1 \mathrm{~h}$ of operating time may be estimated at over $2000 \mathrm{~mL}$ for the proposed configurations. For a typical laparoscopic operation with four ports, this translates to the same volume as 4 two-liter bottles of soda, potentially containing aerosolized contaminants, being released into the OR environment from the patient's surgical cavity. Instrument insertion and removal events (conservatively estimated at 6 events/h in the OR) appear to release additional potentially harmful gas volumes equal to 1-2 cans of twelve ounce sodas [43]. Using an average of 6 instrument removals per hour, results from this study might then suggest a range of $324-684 \mathrm{~mL}$ of potentially contaminated gases released during $1 \mathrm{~h}$ of surgery (i.e. roughly one or two $12 \mathrm{oz}$ cans of soda).
There are opportunities to stop the leakage of gases during laparoscopic surgery in order to protect the OR team from potential viral exposures. This experiment demonstrates that both dynamic instrument interaction with the trocar and instrument insertion and removal will contribute leaked gas into the operating room. Concerns for exposing clinical personnel to COVID-19 significantly reduced elective surgery around the globe for months while COVID restrictions were put in place, which had huge economic impact to hospitals across the world; Ascension, which has 150 hospitals, posted a loss of $\$ 2.7$ billion in the first quarter [45]. Products that allow for reduction of laparoscope and laparoscopic instrument removal would eliminate the need to remove the scope and intstruments during surgery and have significant impact on decreasing gas leakage. Additionally, modifications to the ports could result in decreasing leakage when the instruments are being used. In light of COVID-19 and the expressed danger that may be inherent in laparoscopic surgery due to aerosolized viral particles, serious consideration should be given to new technologies or strategies that minimize risk to the clinicians caring for these patients.

\section{Compliance with ethical standards}

Disclosures Dr. John M. Uecker reports other from ClearCam Inc, outside the submitted work; Mr. Austin Fagerberg and Mr. Naser Ahmad reports personal fees from ClearCam Inc, outside the submitted work; Mr. Mitchell Gilkey and Dr. Christopher R. Idelson reports personal fees and other from ClearCam Inc, outside the submitted work; $\mathrm{Mr}$. Alexander Cohen and Dr. Farshid Alembeigi has nothing to disclose.

\section{References}

1. Burrer SL, de Perio MA, Hughes MM, Kuhar DT, Luckhaupt SE, McDaniel CJ, Porter RM, Silk B, Stuckey MJ, Walters M (2020) Characteristics of Health Care Personnel with COVID19-United States, February 12-April 9, 2020. MMWR Morb Mortal Wkly Rep 69:477-481. https://doi.org/10.15585/mmwr. mm6915e6

2. Francis N, Dort J, Cho E, Feldman L, Keller D, Lim R, Mikami D, Phillips E, Spaniolas K, Tsuda S, Wasco K, Arulampalam T, Sheraz M, Morales S, Pietrabissa A, Asbun H, Pryor A (2020) SAGES and EAES recommendations for minimally invasive surgery during COVID-19 pandemic. Surg Endosc 34:2327-2331. https://doi.org/10.1007/s00464-020-07565-w 
3. COVID-19: Recommendations for Management of Elective Surgical Procedures. https://www.facs.org/covid-19/clinical-guidance/ elective-surgery. Accessed 6 Jun 2020

4. Porter J, Blau E, Gharagozloo F, Martino M, Cerfolio R, Duvvuri U, Caceres A, Badani K, Bhayani S, Collins J, Coelho R, Rocco B, Wiklund P, Nathan S, Parra-Davila E, Ortiz-Ortiz C, Maes K, Dasgupta P, Patel V (2020) Society of robotic surgery review: recommendations regarding the risk of COVID-19 transmission during minimally invasive surgery. BJU Int. https://doi.org/10.1111/ bju. 15105

5. COVID-19: Joint Statement on Minimally Invasive Gynecologic Surgery - AAGL » AAGL. https://www.aagl.org/news/covid -19-joint-statement-on-minimally-invasive-gynecologic-surge ry/?mkt_tok=eyJpIjoiTWpnek9URTROemd4WldKayIsInQiOiJH eWRYUWVwVHhORmx0Smpod3pOc1RxT2pqN05HbFFM b0tWVjlJZDhMcHplUm9uOGMyTGZNcW1uelNGaFwvSitYQ nY1a2EyVzV0QnAyRINlcURnQVwvTzZcL3oraGt3alVIUD k4UjNnd01cL3Z1T212cVwvenlUTW82SExmM3hlR0NPakN ZIn0\%3D. Accessed 6 Jun 2020

6. Kimmig R, Verheijen RHM, Rudnicki M (2020) Robot assisted surgery during the COVID-19 pandemic, especially for gynecological cancer: a statement of the society of European robotic gynaecological surgery (SERGS). J Gynecol Oncol. https://doi. org/10.3802/jgo.2020.31.e59

7. ESGE Recommendations for Gynaecological Endoscopic Surgery for COVID-19 Outbreak

8. EAU Robotic Urology Section (ERUS) guidelines during COVID19 emergency I Uroweb. https://uroweb.org/eau-robotic-urolo gy-section-erus-guidelines-during-covid-19-emergency/?mkt tok=eyJpIjoiTWpnek9URTROemd4WldKayIsInQiOiJHeWRYU WVwVHhORmx 0Smpod3pOc1RxT2pqN05HbFFMb0tWV jlJZDhMcHplUm9uOGMyTGZNcW1uelNGaFwvSitYQnY1a2 EyVzV0QnAyR1NlcURnQVwvTzZcL3oraGt3alVlUDk4UjN nd0lcL3Z1T212cVwvenlUTW82SExmM3hlR0NPakNZIn0\%3D. Accessed 6 Jun 2020

9. Forrester JD, Nassar AK, Maggio PM, Hawn MT (2020) Precautions for operating room team members during the COVID19 pandemic. J Am Coll Surg 230:1098-1101. https://doi. org/10.1016/j.jamcollsurg.2020.03.030

10. Mintz Y, Arezzo A, Boni L, Chand M, Brodie R, Fingerhut A (2020) A low cost, safe and effective method for smoke evacuation in laparoscopic surgery for suspected coronavirus patients. Ann Surg. https://doi.org/10.1097/sla.0000000000003965

11. Vigneswaran Y, Prachand VN, Posner MC, Matthews JB, Hussain M (2020) What is the appropriate use of laparoscopy over open procedures in the current COVID climate. J Gastrointest Surg. 10.1007/s11605-020-04592-9

12. Li Y, Leung GM, Tang JW, Yang X, Chao CYH, Lin JZ, Lu JW, Nielsen PV, Niu J, Qian H, Sleigh AC, Su HJJ, Sundell J, Wong TW, Yuen PL (2007) Role of ventilation in airborne transmission of infectious agents in the built environment-a multidisciplinary systematic review. Indoor Air 17:2-18

13. Garden JM, Kerry O'Banion M, Bakus AD, Olson C (2002) Viral disease transmitted by laser-generated plume (aerosol). Arch Dermatol 138:1303-1307. https://doi.org/10.1001/archd erm.138.10.1303

14. AA Aliabadi SRKBSG (2011) Preventing airborne disease transmission: review of methods for ventilation Design in Health Care Facilities. Adv Prev Med 2011:124064. https://doi. org/10.4061/2011/124064

15. Tellier R, Li Y, Cowling BJ, Tang JW (2019) Recognition of aerosol transmission of infectious agents: a commentary. BMC Infect Dis 19:101

16. Tang JW, Li Y, Eames I, Chan PKS, Ridgway GL (2006) Factors involved in the aerosol transmission of infection and control of ventilation in healthcare premises. J Hosp Infect 64:100-114
17. Jewett DL, Heinsohn P, Bennett C, Rosen A, Neuilly C (1992) Blood-containing aerosols generated by surgical techniques: a possible infectious hazard. Am Ind Hyg Assoc J 53:228-231. https://doi.org/10.1080/15298669291359564

18. Kwak HD, Kim SH, Seo YS, Song KJ (2016) Detecting hepatitis B virus in surgical smoke emitted during laparoscopic surgery. Occup Environ Med 73:857-863. https://doi.org/10.1136/oemed -2016-103724

19. Weyandt GH, Tollmann F, Kristen P, Weissbrich B (2011) Low risk of contamination with human papilloma virus during treatment of condylomata acuminata with multilayer argon plasma coagulation and CO 2 laser ablation. Arch Dermatol Res 303:141-144. https://doi.org/10.1007/s00403-010-1119-3

20. Resources for Smoke \& Gas Evacuation During Open, Laparoscopic, and Endoscopic Procedures-SAGES. https://www. sages.org/resources-smoke-gas-evacuation-during-open-lapar oscopic-endoscopic-procedures/?mkt_tok=eyJpIjoiTWpnek9 URTROemd4WldKayIsInQiOiJHeWRYUWVwVHhORmx 0Smpod3pOc1RxT2pqN05HbFFMb0tWVjlJZDhMcHplUm9u OGMyTGZNcW1uelNGaFwvSitYQnY1a2EyVzV0QnAyRINlc URnQVwvTzZcL3oraGt3alVIUDk4UjNnd01cL3Z1T212cV wvcnlUTW82SExmM3hlR0NPakNZIn0\%3D. Accessed 6 Jun 2020

21. Ferenczy A, Bergeron C, Richart RM (1990) Human papillomavirus DNA in $\mathrm{CO} 2$ laser-generated plume of smoke and its consequences to the surgeon. Obstet Gynecol 75:114-118. https://doi. org/10.1016/0090-8258(89)90871-8

22. Manson LT, Damrose EJ (2013) Does exposure to laser plume place the surgeon at high risk for acquiring clinical human papillomavirus infection? Laryngoscope 123:1319-1320

23. Ulmer B (1998) Occupational Safety and Health Administration acts on guidelines for electrosurgical smoke. AORN J 67:12441245. https://doi.org/10.1016/S0001-2092(06)62612-4

24. Alp E, Bijl D, Bleichrodt RP, Hansson B, Voss A (2006) Surgical smoke and infection control. J Hosp Infect 62:1-5

25. Bendifallah S, Salakos E, Naoura I, Aristizabal P, Furet E, Zilberman S, Ballester M, Darai E (2018) Prospective, randomized comparison of the use of FloShield Air System ${ }^{\circledR}$ versus the reference technique (water + povidone-iodine solution) during gynecologic endoscopic surgery to evaluate the operative lens vision quality. Surg Endosc 32:1593-1599. https://doi.org/10.1007/s0046 4-017-5642-6

26. Workman AD, Welling DB, Carter BS, Curry WT, Holbrook EH, Gray ST, Scangas GA, Bleier BS (2020) Endonasal instrumentation and aerosolization risk in the era of COVID-19: simulation, literature review, and proposed mitigation strategies. Int Forum Allergy Rhinol. https://doi.org/10.1002/alr.22577

27. Gallo O, Locatello LG (2020) Laser-assisted head and neck surgery in the COVID-19 pandemic: controversial evidence and precautions. Head Neck. https://doi.org/10.1002/hed.26271

28. Liu Y, Song Y, Hu X, Yan L, Zhu X (2019) Awareness of surgical smoke hazards and enhancement of surgical smoke prevention among the gynecologists. J Cancer 10:2788-2799

29. Fitzgerald JEF, Malik M, Ahmed I (2012) A single-blind controlled study of electrocautery and ultrasonic scalpel smoke plumes in laparoscopic surgery. Surg Endosc 26:337-342. https ://doi.org/10.1007/s00464-011-1872-1

30. Hwan Choi S, Gyun Kwon T, Kwang Chung S, Kim T-H (2013) Surgical smoke may be a biohazard to surgeons performing laparoscopic surgery. https://doi.org/10.1007/s00464-014-3472-3

31. Transmission of COVID-19. https://www.ecdc.europa.eu/en/covid -19/latest-evidence/transmission. Accessed 29 Aug 2020

32. Memoli MJ, Czajkowski L, Reed S, Athota R, Bristol T, Proudfoot K, Fargis S, Stein M, Dunfee RL, Shaw PA, Davey RT, Taubenberger JK (2015) Validation of the wild-type influenza a human challenge model H1N1pdMIST: an A(H1N1) pdm09 dose-finding 
investigational new drug study. Clin Infect Dis 60:693-702. https ://doi.org/10.1093/cid/ciu924

33. Pujadas E, Chaudhry F, McBride R, Richter F, Zhao S, Wajnberg A, Nadkarni G, Glicksberg BS, Houldsworth J, Cordon-Cardo C (2020) SARS-CoV-2 viral load predicts COVID-19 mortality. Lancet Respir Med. https://doi.org/10.1016/s2213-2600(20)30354 $-4$

34. SARS-CoV-2 viral load and the severity of COVID-19 - CEBM. https://www.cebm.net/covid-19/sars-cov-2-viral-load-and-theseverity-of-covid-19/. Accessed 29 Aug 2020

35. Jacobs VR, Morrison JE, Mettler L, Mundhenke C, Jonat W (1999) Measurement of CO2 hypothermia during laparoscopy and pelviscopy: how cold it gets and how to prevent it. J Am Assoc Gynecol Laparosc 6:289-295. https://doi.org/10.1016/ S1074-3804(99)80063-2

36. Stotz L, Joukhadar R, Hamza A, Thangarajah F, Bardens D, Juhasz-Böss I, Solomayer EF, Radosa MP, Radosa JC (2018) Instrument usage in laparoscopic gynecologic surgery: a prospective clinical trial. Arch Gynecol Obstet 298:773-779. https://doi. org/10.1007/s00404-018-4867-5

37. Sutton E, Youssef Y, Meenaghan N, Godinez C, Xiao Y, Lee T, Dexter D, Park A (2010) Gaze disruptions experienced by the laparoscopic operating surgeon. Surg Endosc 24:1240-1244. https ://doi.org/10.1007/s00464-009-0753-3

38. Geryane MH, Hanna GB, Cuschieri A (2004) Time-motion analysis of operation theater time use during laparoscopic cholecystectomy by surgical specialist residents. Surg Endosc 18:1597-1600. https://doi.org/10.1007/bf02637127

39. Altintoprak F, Akin E, Gundogdu K, Dikicier E (2018) Laparoscopic inguinal hernia repair: technical details, pitfalls and current results. In: Hernia Surgery and Recent Developments. InTech
40. Allers JC, Hussein AA, Ahmad N, Cavuoto L, Wing JF, Hayes RM, Hinata N, Bisantz AM, Guru KA (2016) Evaluation and impact of workflow interruptions during robot-assisted surgery. Urology 92:33-37. https://doi.org/10.1016/j.urology.2016.02.040

41. Lawrentschuk N, Fleshner N, Bolton D (2010) Laparoscopic lens fogging: a review of etiology and methods to maintain a clear visual field. J Endourol 24:905-913

42. Manning T, Perera M, Chistidis D, Kinnear N (2017) Visual occlusion during minimally invasive surgery: a contemporary review of methods to reduce laparoscopic and robotic lens fogging and other sources of optical loss. J Endourol 31:327-333

43. Yong N, Grange P, Eldred-Evans D (2016) Impact of laparoscopic lens contamination in operating theaters: a study on the frequency and duration of lens contamination and commonly utilized techniques to maintain clear vision. Surg Laparosc Endosc Percutaneous Tech 26:286-289. https://doi.org/10.1097/SLE.0000000000 000289

44. Russell RCG (1993) General surgery: biliary surgery. Br Med J 307:1266-1269

45. Providence, Ascension post losses in Q1 as COVID-19 causes drop in patient admissions I FierceHealthcare. https://www.fierc ehealthcare.com/hospitals-health-systems/providence-ascensionpost-losses-q1-as-covid-19-causes-drop-patient. Accessed 10 Jun 2020

Publisher's Note Springer Nature remains neutral with regard to jurisdictional claims in published maps and institutional affiliations. 